\title{
Role of Transporters in Ocular Drug Delivery System
}

\author{
Ashim K. Mitra ${ }^{1,2}$ \\ Received January 26, 2009; accepted February 20, 2009; published online March 17, 2009
}

A number of sight threatening diseases affect the anterior and posterior segments of the eye. Various therapeutic agents have been discovered to treat these disorders, but the challenge still lies in effectively delivering these drugs to the eye, primarily due to the unique anatomy and physiology of the ocular structures. Topical mode of administration is the most preferred and convenient delivery method, but ocular bioavailability of most topically applied drugs is less than $5 \%$. Drug loss is primarily due to precorneal factors such as solution drainage, blinking, tear turnover rate and absorption by adjacent tissues i.e., conjunctiva, resulting in the loss of drug to systemic circulation. Low ocular bioavailability has also been attributed to the lipoidal nature of the corneal epithelium and the water-laden stroma which act as rate limiting barriers for hydrophilic and lipophilic molecules, respectively. In addition, the physicochemical properties of the drug itself limit its permeability across ocular barriers.

Recently, transporter-targeted prodrug approach has received significant attention and a number of membrane transporters have been discovered in various ocular tissues such as cornea, conjunctiva and retina. These transporters are involved in the translocation of essential nutrients and xenobiotics across biological membranes. Ocular transporters include carriers for peptides, amino acids, glucose, lactate and nucleosides/nucleobases and are primarily localized on the epithelia of cornea, conjunctiva and retina. Prodrugs or analogues designed to target these transporters can significantly enhance the absorption of poorly permeating parent drug. Both solubility and membrane permeability can be improved by proper selection of the promoiety. These prodrugs are recognized by the membrane transporters as substrates and are translocated across the epithelia. Subsequently, the prodrugs are enzymatically cleaved to release the parent drug and the ligand which in most cases is a nutrient and thereby non-toxic. Amongst various nutrient transporters that are expressed on the corneal epithelium, the peptide and amino acid transporters have been classified as high capacity low affinity transporters. The current theme section contains an article, which deals with improving the

\footnotetext{
${ }^{1}$ University of Missouri-Kansas City, Kansas City, Missouri, USA.

${ }^{2}$ To whom correspondence should be addressed. (e-mail: mitraa@umkc.edu)
}

corneal permeability of a poorly permeable hydrophilic drug, acyclovir (ACV), for the treatment of herpetic keratitis, by chemically derivatizing it into a prodrug (L-aspartate-acyclovir, L-Asp-ACV). L-Asp-ACV was shown to be a substrate of the corneal $\mathrm{B}^{0,+}$ amino acid transporter and it resulted in a fourfold increase in the transcorneal permeability of ACV. However, D-Asp-ACV did not increase the corneal permeability relative to $\mathrm{ACV}$, suggesting that the L-form of the amino acid is necessary for transporter recognition. Hence, targeting such nutrient transporters on the corneal epithelium is a viable strategy to enhance corneal permeability of hydrophilic therapeutic agents (1).

In addition to the tight junctions expressed by the corneal epithelium, more recently, efflux pumps such as P-glycoprotein (P-gp) have also shown to play a role in restricting ocular bioavailability. Upon instillation of eye drops, the majority of the dose is lost due to drug solution drainage, continuous turnover of the tear film, blinking and absorption by adjacent tissues resulting in drug loss to systemic circulation. Under normal physiological conditions, the volume of the precorneal tear film is about $10 \mu \mathrm{l}$ with an average tear turnover rate of $1.2 \mu \mathrm{l} / \mathrm{min}$. There is instant dilution of the drug by the tear flow upon instillation, with rapid decrease in drug concentration because of continuous turn over. Irritation and external stimuli could further accelerate drug loss from the precorneal area. Consequently, the concentration of the drug in the precorneal area decreases significantly which consequently brings the efflux transporters on the corneal epithelium into play. Apart from the pre-corneal factors, low ocular bioavailability so far has been attributed to the inability of molecules to cross the lipoidal membrane until it was recently shown that P-gp in the corneal epithelium can act as a barrier to in vivo drug absorption though cornea. Multidrug resistance associated protein-2 (MRP2) has also been shown to be functionally active in rabbit and human corneal epithelial cells. An article from our research group in this theme section shows the functional activity of MRP-2 on the rabbit cornea and delineates the involvement of both P-gp and MRP-2 in effluxing a model substrate, erythromycin. The study also discusses the strategy of using therapeutically relevant inhibitors, which can not only inhibit efflux but at the same time can also aid in the treatment regimen. The current article shows inhibition of erythromycin efflux when co-administered along with corticosteroids, which are potential anti-inflammatory agents, thus improving diseases prognosis. 
Depending on the disease condition being treated, other potential therapeutic inhibitors could be used adopting a similar strategy of efflux modulation (2).

Apart from the corneal route of absorption for drugs administered topically, the conjunctiva constitutes an important non-corneal route of absorption for delivery to the posterior segment. Topical mode of administration for delivery to the retina offers a great opportunity, but is limited by the highly lipoidal corneal epithelium (for absorption of drugs across cornea, corneal route) and by the blood vessels and lymphatics of the conjunctiva, draining drugs into the systemic circulation (for absorption of drugs across conjunctiva, non-corneal route). But in both cases, if a sufficiently large amount of drug is made available across the barrier, then therapeutic concentration levels reaching the retina is a possibility. In this regard, we have two research articles from Drs. Ganapathy and Majumdar's group included in the theme section. Dr. Ganapathy's group has identified and functionally characterized a high affinity transporter for opioid peptides on rabbit conjunctival cells. Exploration of the transport systems in the conjunctiva can pave the way for delivery of drug molecules to the back of the eye, thereby bypassing the entire anterior segment. It will be interesting to see if non-opioid peptides will also be transported across the conjunctiva by the transporter that is currently identified. If true, then as discussed in the article, it will broaden the scope of the transport process for ocular delivery for a wide range of peptidomimetic drugs. Similarly Dr. Majumdar's group reports the physicochemical properties and permeability across various ocular tissues for a novel flavanoid, hesperidin, which holds potential in treating age-related macular degeneration, cataract and diabetic retinopathy. The article suggests that stable ophthalmic formulations can be made with sufficient permeability across the cornea, but again further investigation for delivery to the back of the eye is warranted (3-4).

Drug delivery to the posterior segment is faced with a lot of challenges. Transport of molecules between the vitreous/retina and systemic circulation is restricted by the blood-retinal barrier, which is made up of retinal pigment epithelium and endothelial cells of the retinal blood vessels. On the other hand, placing the drug directly in the vitreous humor by intravitreal injections is associated with several problems such as retinal detachment and endophthalmitis. Hence, delivery of drugs via the periocular route offers a better alternative. For posterior segment diseases which require sustained drug levels for a long period of time, sustained release nanoparticulate systems via the periocular route offers hope. An article in the theme section from Dr. Eddelhauser's group discusses transscleral permeability of free drug doxorubicin, PLGA nanoparticles of doxorubicin and a liposomal product of doxorubicin for treating retinoblastoma. The current treatment option is to deliver these anti-cancer drugs systemically leading to severe side-effects. But results from this study show that periocular chemotherapy for retinoblastoma for chemoreduction is feasible. But precaution must be taken such that the release of drug from the formulation is not washed out due to blood vessels and lymphatics of conjunctiva and choroid. It is one of the current challenges which still need to be tackled. So, the future research should focus on decreasing the wash-out of drugs by the conjunctival and choroidal circulation, if not completely eliminated. Since periocular administration has emerged as a promising route for noninvasive delivery to the posterior segment diseases, it would be critical to understand and evaluate formulation and physiological variables for the development of a successful transscleral sustained release delivery system (5).
Even though there are problems associated with intravitreal injections, it is still a viable option for drugs which tend to have longer vitreal half-lives. Vitreal half-life is an important variable which determines the frequency of intravitreal dosing. Drugs with short vitreal half-lives are eliminated rapidly, requiring frequent injections which may lead to poor patient compliance. But currently, there is not a proper model available which can predict the vitreal half-lives of a wide range of drug molecules, without actually having to determine them in vivo. The article by Dr. Kompella's group, tries to develop a model for a wide range of drug molecules belonging to different categories, taking into consideration parameters such as $\log \mathrm{P}, \log \mathrm{D}, \log \mathrm{MW}$ and dose number (dose/solubility). The article discusses the importance of one or more parameters in the successful development of a model for a particular class of drug. This model could be widely used by scientists and clinicians to predict the vitreal half-life and decide whether the benefits of the therapy could out-weigh the risks associated with intravitreal injections (6).

Finally, we have also included a review article from our group which focuses on the barriers, current strategies and developments in the field of ocular delivery. The article emphasizes some of the recent advances in the field of design of implants and gene delivery systems. Successful ocular drug delivery has been considered a major challenge by scientists in the field. Understanding the ocular barriers such as anatomical and physiological barriers including efflux of drugs has proven crucial to optimize drug delivery to the eye. Role of nutrient transporters and receptors in delivering the drugs to target tissue has gathered a lot of attention recently. Targeting the nutrient transporters can help in overcoming the tight junctions and also bypass efflux simultaneously, thereby significantly increasing ocular bioavailability. Recent developments, especially over the last decade, in the field of drug delivery, utilizing nanocarriers are exciting and promising as well. These nanocarriers can target as well as sustain drug release, which is crucial for diseases affecting the back of the eye. Overall, current momentum in the field of ocular drug delivery holds a great future for the development of targeted, non invasive and controlled release formulations for the treatment of vision threatening ocular diseases in both the anterior and posterior segments (7).

\section{About The Guest Editor}

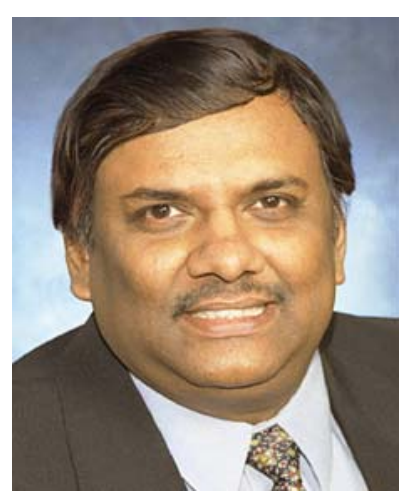

Dr. Ashim K. Mitra was recently named one of two recipients for the 2007 ARVO/Pfizer Ophthalmics Translational Award. He is the Vice Provost for Interdisciplinary Research and Director for Translational Research at University of Missouri-Kansas City, 
School of Medicine. Dr. Mitra is also a Curators' Professor and Chairman of Pharmaceutical Sciences at the School of Pharmacy. He obtained his Ph.D. in Pharmaceutical Chemistry, from University of Kansas in 1983. He joined his current position after serving as an Associate Professor in the School of Pharmacy at Purdue University, West Lafayette, IN, USA. He has over 25 years of experience in the field of ocular drug delivery and disposition. He authored and co-authored over 200 refereed articles, published in various international journals. He also served as the editor of 1993 and 2003 editions of the well-known text book, Ophthalmic Drug Delivery Systems and has authored or co-authored over twenty chapters for various texts relating to ocular drug delivery. His work has also been presented at several universities, pharmaceutical companies and scientific organizations, worldwide. He serves on the editorial advisory board for Bentham Science Publishers, International Journal of Pharmaceutics, Clinical Research and Regulatory Affairs, Current Eye Research and Journal of Ocular Pharmacology and Therapeutics. He also served as a regular member of Pharmacology Study Section at National Institutes of Health (NIH). Dr. Mitra is the recipient of a number of research awards from the NIH, the American Association of Pharmaceutical Scientists (AAPS), the American Association of Colleges of Pharmacy (AACP) and numerous pharmaceutical organizations. He is the inventor of two US patents in the field of drug delivery with one patent focused on ocular drug delivery.

\section{Interview Questions for Dr. Ashim Mitra}

1. What do you think holds the key to your success as a pharmaceutical scientist?

Response: The keys to my success as pharmaceutical scientist started with very strong science education in secondary schools in India as well as strong background in pharmaceutical sciences and research from my under graduate and masters degree training from Jadavpur University, India. This background propelled me to become a graduate student in the pharmaceutical chemistry department at the University of Kansas. Excellent training from my mentors and the environment at Kansas gave me the necessary impetus to join academia and be a strong independent pharmaceutical scientist. I have also been fortunate enough to attract outstanding graduate and post doctoral associates at both Purdue University and the University of Missouri-Kansas City, which also holds the keys to my becoming a successful pharmaceutical scientist.

2. What do you consider to be your key research accomplishments?

Response: My research accomplishments over the last 25 years after receiving a doctoral degree in pharmaceutical chemistry can best be summarized in the following research areas.

Transporter targeted delivery of prodrug: My group has exploited the idea of conjugating nutrients like amino acids or peptides to the drug moiety (prodrug), thereby, targeting a nutrient transporter, which recognizes them as substrates and translocates them across the epithelium, significantly enhancing the absorption of poorly permeating parent drug. After translocation, the prodrugs are enzymatically cleaved to release the parent drug and free ligand, which in most cases is a nutrient and is non-toxic. Hence, my research team synthesized a series of water soluble dipeptide prodrugs of acyclovir and ganciclovir targeting the peptide transporter system on the corneal epithelium to improve ocular bioavailability. These prodrugs exhibited excellent solubility, solution stability and transcorneal permeability. In addition, they proposed higher anti-viral efficacy and lower cytotoxicity in comparison to trifluorothymidine. Prodrug strategy has been also employed successfully in my laboratory to circumvent P-glycoprotein mediated efflux. Such a strategy has previously resulted in three-fold increase in corneal permeation of quinidine, a model P-gp substrate. Success of the above strategy depends on the properties of the influx transporters, mainly capacity and substrate affinity.

Development of ocular microdialysis technique: My research group has pioneered the use of a novel microdialysis technique to study the ocular disposition and pharmacokinetics of antiviral, antimicrobial, antitumor and other anti-infective agents. Prior to the development of this technique, study of ocular pharmacokinetics of drugs in an animal model was extremely difficult. Conventional pharmacokinetic studies required sacrificing at least six animals per time point. Moreover, using such a large number of animals caused extreme variability in the data, often rendering statistical interpretation of the results, difficult. On the other hand, microdialysis has been proven to be beneficial over conventional sampling techniques in determining ocular pharmacokinetics by both reducing the number of subjects and providing statistically robust data. This technique has been successfully developed to monitor drug concentrations in the aqueous and vitreous humor of rabbits. My laboratory has also succeeded in developing a conscious microdialysis rabbit model, which helps monitor the drug concentrations in vitreo-retinal spaces, released from implants and novel polymeric sustained-release formulations developed in the laboratory.

Development of novel pentablock polymer for sustained release of drugs to vitreo-retinal spaces: Many sight threatening disease states such as cytomegaloviral (CMV) retinitis, age-related macular degeneration (AMD), proliferative and diabetic vitreoretinopathy and endophthalmitis exist in the posterior segment of the eye with retina being the primary target for most of the diseases. Topical, systemic and intravitreal mode of administration suffers from major drawbacks thereby limiting their application. This current challenge in ophthalmic drug delivery has been met by my research group by finding an effective drug delivery technology to circumvent the ocular barriers without causing significant patient discomfort or alterations to the protective ocular mechanisms. My research team has conceptualized administering a sustained release formulation through the periocular route utilizing nanoparticles prepared from novel pentablock polymers and suspending them in a thermosensitive gel. Nanoparticles suspended in a thermosensitive gel minimized the burst release of drugs which is a common problem encountered with conventional nanoparticles. Such novel technology when injected periocularly, results in a prolonged duration of action thereby eliminating the need for repeated administration. In future, we will focus on more non-invasive drug delivery systems through the periocular route which can provide constant drug levels in the retina for long period of time after single administration. 
Formulation of a mixed micellar formulation of calcineurin inhibitor for keratoconjunctivitis sicca: My research group in collaboration with Lux Biosciences Inc. has broken new grounds in developing a novel, clear mixed micellar formulation for voclosporin. Voclosporin is an analog of cyclosporine, which is four times more potent and patient compliant than Restasis ${ }^{\circledR}$ for the treatment of dry eye syndrome. But as a substance, voclosporin is very hydrophobic and posed a major challenge for it to be formulated into eye drops. The challenge was successfully met by my group which developed a clear, mixed micellar solution of voclosporin comprised of amphiphilic polymers such as Vit E TPGS and Octoxynol-40, mixed at optimum ratios. The resulting formulation exhibited superior tolerability in rabbits with mean irritation scores being highly significant between groups treated with Restasis ${ }^{\circledR}$ and the novel mixed micellar formulation. Moreover, ocular distribution studies showed a significant concentration of the drug achieved in the posterior segment tissues such as retinachoroid and optic nerve. So, this strategy has also opened the gateway to deliver drugs to the back of the eye from topical administration.

\section{What was turning point in your career?}

Response: As a graduate student I was fascinated by the work of Higuchi and Stella on prodrugs in the 1970s. My own research was directed towards improving drug absorption through the cornea. Also, I was interested in developing antiviral therapies. Therefore, based on my training and research interest I thought that application of prodrug principles to antiviral agents may open up novel prodrug strategies for the treatment of ocular viral infections. Therefore, I wrote my first NIH grant within a few months after my graduation from the University of Kansas. This initial grant application received excellent reviews from the NIH study section and was funded. This accomplishment led me to the path of application of physical chemical strategies to improving drug absorption and overall bioavailability particularly to the eye. I believe that this must be the turning point in my carrier. This accomplishment at such an early stage in my career gave me the direction upon which I built my research program over the career within the last 25 years.

4. Who are the individuals who had the greatest influence on your research career?

Response: Although several people influenced my research career, I must put Thomas Mikkelson my PhD mentor at the University of Kansas at the top as being the most influential. He introduced me to the field of ophthalmic drug delivery research. I was his first graduate student and I developed various techniques particularly animal ocular pharmacokinetic and pharmacodynamic work during my work as graduate student at Kansas. My other graduate committee members particularly Takeru Higuchi, Valentino Stella provided me with strong background in physical chemical sciences. Also, I was very much influenced by the work of Joseph Robinson who pioneered the field of ocular biopharmaceutics and pharmacokinetics. Therefore a strong background in physical, chemical and biological sciences allowed me to become a strong academic pharmaceutical scientist.

5. Pharmaceutical scientists are faced with the dilemma of having to publish in biomedical or basic science journals. Does it mean cutting edge science will not likely be featured in the Pharmaceutical Research?
Response: I consider pharmaceutical science particularly the field of pharmaceutics to be an applied science where basic science principles are applied to solve biological problems. For that reason one must have very good training in physical, chemical and mathematical sciences to understand and research complex biological phenomena. As a pharmaceutical scientist I believe that it is my responsibility to move the frontier by publishing cutting edge science in both biomedical and basic science journals. By looking at my publication record it is apparent that I have published my majority of articles in pharmaceutical science journals such as Pharmaceutical Research, International Journal of Pharmaceutics, Molecular Pharmaceutics and Journal of Pharmaceutical Sciences. However, ocular drug delivery has become an interesting field for ophthalmologists, veterinary ophthalmologists and pharmacologists. Therefore in the last 10 years, I started publishing more and more in the top vision research journals such as Investigative Ophthalmology and Visual Science, Current Eye Research, and Journal of Ocular Pharmacology and Therapeutics. By publishing in both types of journals, gave my research the maximum exposure to my colleagues in academia, industry and government organizations.

6. What is the key to developing successful collaborative relationship?

Response: Pharmaceutical research more and more is becoming highly multidisciplinary in nature. Therefore, one must establish collaborative relationships with experts in other fields who can provide a well rounded research project with key out comes. From the beginning of my career I realized the importance of collaborating with virologists, bacteriologists and clinicians. The field of translational research where research from bench is translated to the bedside of a patient holds the key to a successful research program of pharmaceutical scientist. I found in my research career that scientist with expertise in various fields are also eager to collaborate with strong basic as well as pharmaceutical scientists. Development of appropriate collaborative programs can enrich and stimulate existing research programs.

7. Where is the place for collaboration with industry in academia?

Response: For over 25 years in my research career I have always attempted to collaborate with industries from two perspectives. One is to find a mutually beneficial research project which is publishable and the other is that it must provide the adequate support in terms of research funding. I was able to publish most, if not all of the industrial research projects. Recently, I was involved with several projects with industry where viable products and novel patentable drug delivery systems could be developed and such systems could be introduced into clinical trials. Sometimes academic researchers can provide unique technologies which could be utilized by the industry to obtain results necessary for the development of a product. In such cases research contract may be a suitable vehicle to undertake industry sponsor research.

\section{What is your philosophy of educating graduate students?}

Response: Graduate students should undergo extensive theoretical and practical training within a normal span of 4-5 years so that they can become independent scientists. I expect all of the graduate students to undertake core courses at both undergraduate and 
graduate levels in order to gain a rounded knowledge in physical, chemical and biological sciences. Therefore, a strong background in chemistry and mathematics must be complimented with biological courses such as molecular biology, cell biology, biochemistry and immunology. Moreover, a graduate student in pharmaceutical sciences must have adequate knowledge in basic pharmaceutical principles such as drug stability, solubility, preformulation and formulation properties as well as pharmacokinetics, pharmacodynamics and drug metabolism. Since the scope of pharmaceutical sciences is rapidly expanding with tremendous growth in the biological arena, it is likely that more and more graduate courses in the biological sciences may be necessary to accomplish the goals of a research project. As such I believe that every graduate student should develop his or her own plan of study based on the requirements within the department as well as the theoretical background necessary to tackle the research project.

9. What are the challenges facing the pharmaceutical scientists?

Response: Pharmaceutical sciences, particularly the field of pharmaceutics and pharmaceutical chemistry in the next decade will face significant challenges from both academic and industrial perspectives. With the rapid expansion of the clinical programs particularly the PharmD programs it will be difficult to train pharmaceutical scientists with a degree from US pharmacy schools. Therefore, it is very likely that most of the pharmaceutical scientists in both academic and industry may come with basic degrees in chemistry, biology and engineering. Some orientation of these scientists to the classic pharmaceutical problems and principles may help in expanding the horizon in this area. Apart from the strong background in physical, chemical and biological sciences pharmaceutical scientists may require some understanding of clinical research, personalized medicine and integration of pharmacogenomics and proteomics in developing individualized medicine. An appreciation for biomarkers and understanding the role of various efflux pumps in drug absorption, distribution, metabolism and excretion may allow them to offer unique perspective in drug therapy. With the downsizing of the so called large traditional pharmaceutical companies it is likely that more job opportunities for the pharmaceutical science graduates may come from small boutique biotechnology as well as generic and contract manufacturing companies. Therefore, this graduate should possess adequate knowledge to tackle large or macromolecular therapeutic agents, their analysis, PK/PD properties and toxicity. In addition to this they also should have knowledge in the preformulation, formulation, and manufacturing of such agents. Moreover they should have training in new and emerging technologies such as LC-MS/MS, RT-PCR, in vivo imaging as well as pharmacogenomics. Therefore, it is very unlikely that a graduate student with a span of 4-5 years in graduate school will master all of these technologies. However, their basic training should be such that they can acquire additional knowledge as required by the job.

\section{REFERENCES}

1. S. Majumdar, T. Hingorani, R. Srirangam, R. S. Gadepalli, J. M. Rimoldi, and M. A. Repka. Transcorneal permeation of L and D aspartate ester prodrugs of acyclovir: delineation of passive diffusion versus transporter involvement. doi:10.1007/s11095-0089730-0

2. S. Hariharan, S. Gunda, G. P. Mishra, D. Pal, and A. K. Mitra. Enhanced corneal absorption of erythromycin by modulating PGlycoprotein and MRP mediated efflux with corticosteroids. doi:10.1007/s11095-008-9741-x

3. S. Majumdar, and R. Srirangam. Solubility, stability, physicochemical characteristics and in vitro ocular tissue permeability of hesperidin: a natural bioflavonoid. doi:10.1007/s11095-008-9729-6

4. S. Ananth, S. Karunakaran, P. M. Martin, C. N. Nagineni, J. J. Hooks, S. B. Smith, P. D. Prasad, and V. Ganapathy. Functional identification of a novel transport system for endogenous and synthetic opioid peptides in the rabbit conjunctival epithelial cell line CJVE. doi:10.1007/s11095-008-9709-x

5. E. S. Kim, C. Durairaj, R. S. Kadam, S. J. Lee, Y. Mo, D. H. Geroski, U. B. Kompella, and H. F. Edelhauser. Human scleral diffusion of anticancer drugs from solution and nanoparticle formulation. doi:10.1007/s11095-009-9835-0

6. C. Durairaj, J. C. Shah, S. Senapati, and U. B. Kompella. Prediction of vitreal half-life based on drug physicochemical properties: quantitative structure-pharmacokinetic relationships (QSPKR). doi:10.1007/s11095-008-9728-7

7. R. Gaudana, J. Jwala, S. H. Boddu, and A. K. Mitra. Recent perspectives in ocular drug delivery. doi:10.1007/s11095-008-9694-0 